\title{
A SELECTION OF 20 CURRENT READINGS ON TOPICS RELATED TO COVID- 19: SINGAPORE EXPERIENCE
}

\author{
Some available as free full-text and some requiring payment
}

Selection of readings made by A/Prof Goh Lee Gan

\section{READING I - COVID- I9 OUTBREAK - ORIGIN, TRANSMISSION AND CLINICAL THERAPIES}

Guo YR(I), Cao QD(2), Hong ZS(3), Tan YY(I), Chen SD(I), Jin HJ(I), Tan KS(4), Wang DY(5), Yan $Y(6)(7)$. The origin, transmission and clinical therapies on coronavirus disease 20 I9 (COVID- I9) outbreak - an update on the status. Mil Med Res. 2020 Mar I3;7(I): I I.

\section{URL:doi: I 0.I I86/s40779-020-00240-0.(Free full text)}

Author information:

(1)Guangdong Provincial Key Laboratory of Biomedical Imaging and Guangdong Provincial Engineering Research Center of Molecular Imaging, Zhuhai, 519000, Guangdong, China. (2)Department of Cardiothoracic Surgery, the Fifth Affiliated Hospital, Sun Yat-Sen University, Zhuhai, 519000, Guangdong, China. (3)Center of Infectious Disease, the Fifth Affiliated Hospital, Sun Yat-Sen University, Zhuhai, 519000, Guangdong, China. (4)Department of Otolaryngology, Yong Loo Lin School of Medicine, National University of Singapore, National University Health System, Singapore, 119228, Singapore. (5)Department of Otolaryngology, Yong Loo Lin School of Medicine, National University of Singapore, National University Health System, Singapore, 119228, Singapore. entwdy@nus.edu.sg. (6)Guangdong Provincial Key Laboratory of Biomedical Imaging and Guangdong Provincial Engineering Research Center of Molecular Imaging, Zhuhai, 519000, Guangdong, China. yanyan35@mail.sysu.edu.cn. (7)Center for Interventional Medicine, the Fifth Affiliated Hospital, Sun Yat-Sen University, Zhuhai, 519000, Guangdong, China. yanyan35@mail.sysu.edu.cn.

\section{$\underline{\text { ABSTRACT }}$}

An acute respiratory disease, caused by a novel coronavirus (SARS-CoV-2, previously known as 2019-nCoV), the coronavirus disease 2019 (COVID-19) has spread throughout China and received worldwide attention. On 30 January 2020, World Health Organisation (WHO) officially declared the COVID-19 epidemic as a public health emergency of international concern. The emergence of SARS-CoV-2, since the severe acute respiratory syndrome coronavirus (SARS-CoV) in 2002 and Middle East respiratory syndrome coronavirus (MERS-CoV) in 2012, marked the third introduction of a highly pathogenic and large-scale epidemic coronavirus into the human population in the twenty-first century.

As of $1^{\text {st }}$ March 2020, a total of 87,137 confirmed cases globally, 79,968 confirmed in China and 7169 outside of China, with 2977 deaths (3.4 percent) had been reported by WHO. Meanwhile, several independent research groups have identified that SARS-CoV-2 belongs to $\beta$-coronavirus, with highly identical genome to bat coronavirus, pointing to bat as the natural host. The novel coronavirus uses the same receptor, angiotensin-converting enzyme 2 (ACE2) as that for SARS-CoV, and mainly spreads through the respiratory tract. Importantly, increasingly evidence showed sustained human-to-human transmission, along with many exported cases across the globe.

The clinical symptoms of COVID-19 patients include fever, cough, fatigue and a small population of patients appeared gastrointestinal infection symptoms. The elderly and people with underlying diseases are susceptible to infection and prone to serious outcomes, which may be associated with acute respiratory distress syndrome (ARDS) and cytokine storm. Currently, there are few specific antiviral strategies, but several potent candidates of antivirals and repurposed drugs are under urgent investigation.

In this review, we summarised the latest research progress of the epidemiology, pathogenesis, and clinical characteristics of COVID-19, and discussed the current treatment and scientific advancements to combat the epidemic novel coronavirus. 


\title{
READING 2 - CAN WE CONTAIN THE COVID- I 9 OUTBREAK WITH SAME MEASURES FOR SARS?
}

\author{
Wilder-Smith A(I), Chiew CJ(2), Lee VJ(3). Can we contain the COVID-I9 outbreak with the same \\ measures as for SARS? Lancet Infect Dis. 2020 May;20(5):e I 02-el 07.
}

\section{URL: doi: 10.10 I6/S I 473-3099(20)30 I 29-8 (Free full text).}

Author information:

(1)London School of Hygiene and Tropical Medicine, London, UK; Heidelberg Institute of Global Health, University of Heidelberg, Heidelberg, Germany; Lee Kong Chian School of Medicine, Singapore, Singapore. Electronic address: annelies.wilder-smith@lshtm.ac.uk. (2)National University Health System, Singapore, Singapore. (3)Saw Swee Hock School of Public Health, National University of Singapore, Singapore, Singapore.

\section{$\underline{\text { ABSTRACT }}$}

The severe acute respiratory syndrome (SARS) outbreak in 2003 resulted in more than 8000 cases and 800 deaths. SARS was eventually contained by means of syndromic surveillance, prompt isolation of patients, strict enforcement of quarantine of all contacts, and in some areas top-down enforcement of community quarantine.

By interrupting all human-to-human transmission, SARS was effectively eradicated. By contrast, by Feb 28, 2020, within a matter of two months since the beginning of the outbreak of coronavirus disease 2019 (COVID-19), more than 82,000 confirmed cases of COVID-19 have been reported with more than 2800 deaths. Although there are striking similarities between SARS and COVID-19, the differences in the virus characteristics will ultimately determine whether the same measures for SARS will also be successful for COVID-19. COVID-19 differs from SARS in terms of infectious period, transmissibility, clinical severity, and extent of community spread.

Even if traditional public health measures are not able to fully contain the outbreak of COVID-19, they will still be effective in reducing peak incidence and global deaths. Exportations to other countries need not result in rapid large-scale outbreaks, if countries have the political will to rapidly implement countermeasures.

\section{READING 3 - WE URGENTLY NEED TO START DEVELOPING AN EXIT STRATEGY}

Petersen E(I), Wasserman S(2), Lee SS(3), Go U(4), Holmes AH(5), Al-Abri S(6), McLellan S(7), Blumberg L(8), Tambyah P(9). Covid- I 9-We urgently need to start developing an exit strategy. Int J Infect Dis. 2020 Apr 29;96:233-239

\section{URL: doi: 10.1016/j.ijid.2020.04.035 (Free full text). Online ahead of print.}

Author information:

(1)Institute for Clinical Medicine, Faculty of Health Sciences, University of Aarhus, Denmark; Department of Molecular Medicine, University of Pavia, Italy; European Society for Clinical Microbiology and Infectious Diseases (ESCMID) Task Force for Emerging Infections. Basel, Switzerland. (2)Division of Infectious Diseases and HIV Medicine, Department of Medicine, Groote Schuur Hospital and University of Cape Town, Cape Town, South Africa. (3)Stanley Ho Centre for Emerging Infectious Diseases, The Chinese University of Hong Kong, Shatin, Hong Kong, SAR China. (4)International Tuberculosis Research Center, Changwon-si, Republic of Korea. (5)National Institute for Health Research Health Protection Research Unit in Healthcare Associated Infections and Antimicrobial Resistance, Imperial College London, Hammersmith Campus, London, United Kingdom. (6)Directorate General for Disease Control and Prevention, Ministry of Health, Oman. (7)Biocontainment Treatment Unit, Biosafety for Research-Related Infectious Pathogens, University of Texas Medical Branch, Galveston, TX, USA. (8)National Institute for Communicable Diseases, A Division of the National Health Laboratory Service, Johannesburg, South Africa. (9)Division of Infectious Disease, Department of Medicine University Medicine Cluster, National University Hospital, Singapore. Electronic address: mdcpat@nus.edu.sg. 


\begin{abstract}
AIM: The purpose of this perspective is to review the options countries have to exit the draconian "lockdowns" in a carefully staged manner.
\end{abstract}

METHODS: Experts from different countries experiencing Corona Virus Infectious Disease 2019 (COVID-19) reviewed evidence and country-specific approaches and the results of their interventions.

RESULTS: Three factors are essential: 1 . Reintroduction from countries with ongoing community transmission; 2 . The need for extensive testing capacity and widespread community testing, and 3. An adequate supply of personal protective equipment, PPE, to protect health care workers. Discussed at length are lifting physical distancing, how to open manufacturing and construction, logistics, and the opening of higher educational institutions and schools. The use of electronic surveillance is considered.

CONCLUSION: Each country should decide on the best path forward. However, we can learn from each other, and the approaches are, in reality, very similar.

\title{
READING 4 - CONNECTING CLUSTERS OF COVID- 19
}

Yong SEF(1), Anderson DE(2), Wei WE(3), Pang J(4), Chia WN(2), Tan CW(2), Teoh YL(5), Rajendram P(6), Toh MPHS(7), Poh C(6), Koh VTJ(6), Lum J(6), Suhaimi NM(6), Chia PY(8), Chen MI(7), Vasoo S(8), Ong B(9), Leo YS(8), Wang L(2), Lee VJM(10). Connecting clusters of COVID- I9: an epidemiological and serological investigation. Lancet Infect Dis. 2020 Apr 2 I:S I473-3099(20)30273-5.

\section{URL: doi: 10.1016/S I473-3099(20)30273-5 (Free full text). Online ahead of print.}

Author information:

(1)Ministry of Health, Singapore; National University Health System, Singapore. (2)Programme in Emerging Infectious Diseases, Duke-National University of Singapore Medical School, Singapore. (3)Ministry of Health, Singapore; National Centre for Infectious Diseases, Singapore. (4)Centre for Infectious Disease Epidemiology and Research, Singapore; Saw Swee Hock School of Public Health, Singapore. (5)Saw Swee Hock School of Public Health, Singapore; Singapore Clinical Research Institute, Singapore; Ministry of Health Office for Healthcare Transformation, Singapore. (6)Ministry of Health, Singapore. (7)National Centre for Infectious Diseases, Singapore; Saw Swee Hock School of Public Health, Singapore. (8)National Centre for Infectious Diseases, Singapore; Tan Tock Seng Hospital, Singapore. (9)Ministry of Health, Singapore; National University Health System, Singapore; National University of Singapore, Singapore. (10)Ministry of Health, Singapore; Saw Swee Hock School of Public Health, Singapore. Electronic address: vernon_lee@moh.gov.sg.

\section{ABSTRACT}

BACKGROUND: Elucidation of the chain of disease transmission and identification of the source of coronavirus disease 2019 (COVID-19) infections are crucial for effective disease containment. We describe an epidemiological investigation that, with use of severe acute respiratory syndrome coronavirus 2 (SARS-CoV-2) serological assays, established links between three clusters of COVID-19.

METHODS: In Singapore, active case-finding and contact tracing were undertaken for all COVID-19 cases. Diagnosis for acute disease was confirmed with RT-PCR testing. When epidemiological information suggested that people might have been nodes of disease transmission but had recovered from illness, SARS-CoV-2 IgG serology testing was used to establish past infection. FINDINGS: Three clusters of COVID-19, comprising 28 locally transmitted cases, were identified in Singapore; these clusters were from two churches (Church A and Church B) and a family gathering. The clusters in Church $A$ and Church B were linked by an individual from Church A (A2), who transmitted SARS-CoV-2 infection to the primary case from Church B (F1) at a family gathering they both attended on Jan 25, 2020. All cases were confirmed by RT-PCR testing because they had active disease, except for A2, who at the time of testing had recovered from their illness and tested 
negative. This individual was eventually diagnosed with past infection by serological testing. ELISA assays showed an optical density of more than 1.4 for SARS-CoV-2 nucleoprotein and receptor binding domain antigens in titres up to 1/400, and viral neutralisation was noted in titres up to $1 / 320$.

INTERPRETATION: Development and application of a serological assay has helped to establish connections between COVID-19 clusters in Singapore. Serological testing can have a crucial role in identifying convalescent cases or people with milder disease who might have been missed by other surveillance methods.

FUNDING: National Research Foundation (Singapore), National Natural Science Foundation (China), and National Medical Research Council (Singapore).

\section{READING 5 - MIGRANT WORKERS AND COVID- I 9 IN SINGAPORE}

Koh D(I)(2). Migrant workers and COVID- 19. Occup Environ Med. 2020 Jun 8:oemed-2020- 106626. doi: I0.I I36/oemed-2020- 106626.

Online ahead of print.

Author information:

(1)Institute of Health Sciences, Universiti Brunei Darussalam, Gadong, Brunei Darussalam david_koh@nuhs.edu.sg. (2)Saw Swee Hock School of Public Health, National University of Singapore, Singapore.

\section{ABSTRACT}

OBJECTIVES: Daily numbers of COVID-19 in Singapore from March to May 2020, the cause of a surge in cases in April and the national response were examined, and regulations on migrant worker accommodation studied.

METHODS: Information was gathered from daily reports provided by the Ministry of Health, Singapore Statues online and a Ministerial statement given at a Parliament sitting on $4^{\text {th }}$ May 2020.

RESULTS: A marked escalation in the daily number of new COVID-19 cases was seen in early April 2020. The majority of cases occurred among an estimated 295,000 low-skilled migrant workers living in foreign worker dormitories. As of $6^{\text {th }}$ May 2020, there were 17,758 confirmed COVID-19 cases among dormitory workers (88 percent of 20,198 nationally confirmed cases). One dormitory housing approximately 13,000 workers had 19.4 percent of residents infected. The national response included mobilising several government agencies and public volunteers. There was extensive testing of workers in dormitories, segregation of healthy and infected workers, and daily observation for fever and symptoms. Twenty-four dormitories were declared as 'isolation areas', with residents quarantined for 14 days. New housing, for example, vacant public housing flats, military camps, exhibition centres, floating hotels have been provided that will allow for appropriate social distancing.

CONCLUSION: The COVID-19 pandemic has highlighted migrant workers as a vulnerable occupational group. Ideally, matters related to inadequate housing of vulnerable migrant workers need to be addressed before a pandemic.

READING 6 - POTENTIAL UTILITIES OF MASK WEARING AND INSTANT HAND HYGIENE

Ma QX(I), Shan H(I), Zhang HL(I), Li GM(I), Yang RM(I), Chen JM(I). Potential utilities of mask-wearing and instant hand hygiene for fighting SARS-CoV-2. J Med Virol. 2020 Mar 3 I:10.1 002/jmv.25805.

URL: doi: 10.1002/jmv.25805 (Free full text).

Online ahead of print. 
Author information:

(1)College of Veterinary Medicine, Qingdao Agricultural University, Qingdao, China.

\section{ABSTRACT}

The surge of patients in the pandemic of COVID-19 caused by the novel coronavirus SARS-CoV-2 may overwhelm the medical systems of many countries. Mask-wearing and handwashing can slow the spread of the virus, but currently, masks are in shortage in many countries, and timely handwashing is often impossible.

In this study, the efficacy of three types of masks and instant hand wiping was evaluated using the avian influenza virus to mock the coronavirus.

Virus quantification was performed using real-time reverse transcription-polymerase chain reaction. Previous studies on mask-wearing were reviewed. The results showed that instant hand wiping using a wet towel soaked in water containing 1.00 percent soap powder, 0.05 percent active chlorine, or 0.25 percent active chlorine from sodium hypochlorite removed 98.36 percent, 96.62 percent, and 99.98 percent of the virus from hands, respectively. N95 masks, medical masks, and homemade masks made of four-layer kitchen paper and one-layer cloth could block 99.98 percent, 97.14 percent, and 95.15 percent of the virus in aerosols.

Medical mask-wearing which was supported by many studies was opposed by other studies possibly due to erroneous judgment.

With these data, we propose the approach of mask-wearing plus instant hand hygiene (MIH) to slow the exponential spread of the virus. This MIH approach has been supported by the experiences of seven countries in fighting against Covid-19.

Collectively, a simple approach to slow the exponential spread of SARS-CoV-2 was proposed with the support of experiments, literature review, and control experiences.

\section{READING 7 - ROLE OF COMMUNITY-WIDE WEARING OF FACE MASK FOR CONTROL OF COVID- 19}

Cheng VC(I), Wong SC(2), Chuang VW(3), So SY(4), Chen JH(4), Sridhar S(5), To KK(5), Chan JF(5), Hung IF(6), Ho PL(5), Yuen KY(7).The role of community-wide wearing of face mask for control of coronavirus disease 2019 (COVID- I9) epidemic due to SARS-CoV-2.J Infect. 2020 Apr 23;8I(I): I07-I4. J Infect. 2020 Apr 23;8I(I): I07-I4.

\section{URL: doi: 10.1016/j.jinf.2020.04.024. Online Epub ahead of print.}

Author information:

(1)Department of Microbiology, Queen Mary Hospital, Hong Kong Special Administrative Region, China; Infection Control Team, Queen Mary Hospital, Hong Kong West Cluster, Hong Kong Special Administrative Region, China. (2)Infection Control Team, Queen Mary Hospital, Hong Kong West Cluster, Hong Kong Special Administrative Region, China. (3)Quality \& Safety Division (Infection, Emergency, and Contingency), Hospital Authority, Hong Kong Special Administrative Region, China. (4)Department of Microbiology, Queen Mary Hospital, Hong Kong Special Administrative Region, China. (5)Department of Microbiology, Li Ka Shing Faculty of Medicine, The University of Hong Kong, Hong Kong Special Administrative Region, China. (6)Department of Medicine, Li Ka Shing Faculty of Medicine, The University of Hong Kong, Hong Kong Special Administrative Region, China. (7)Department of Microbiology, Li Ka Shing Faculty of Medicine, The University of Hong Kong, Hong Kong Special Administrative Region, China. Electronic address: kyyuen@hku.hk. 


\section{ABSTRACT}

BACKGROUND: Face mask usage by the healthy population in the community to reduce risk of transmission of respiratory viruses remains controversial. We assessed the effect of community-wide mask usage to control coronavirus disease 2019 (COVID-19) in Hong Kong Special Administrative Region (HKSAR).

METHODS: Patients presenting with respiratory symptoms at outpatient clinics or hospital wards were screened for COVID-19 per protocol. Epidemiological analysis was performed for confirmed cases, especially persons acquiring COVID-19 during mask-off and mask-on settings. The incidence of COVID-19 per million population in HKSAR with community-wide masking was compared to that of non-mask-wearing countries which are comparable with HKSAR in terms of population density, healthcare system, BCG vaccination and social distancing measures but not community-wide masking. Compliance of face mask usage in the HKSAR community was monitored.

FINDINGS: Within first 100 days (31 $1^{\text {st }}$ December 2019 to $8^{\text {th }}$ April 2020), 961 COVID-19 patients were diagnosed in HKSAR. The COVID-19 incidence in HKSAR (129.0 per million population) was significantly lower $(\mathrm{p}<0.001)$ than that of Spain (2983.2), Italy (2250.8), Germany (1241.5), France (1151.6), U.S. (1102.8), U.K. (831.5), Singapore (259.8), and South Korea (200.5).

The compliance of face mask usage by HKSAR general public was 96.6 percent (range: 95.7 percent to 97.2 percent). We observed 11 COVID-19 clusters in recreational 'mask-off' settings compared to only three in workplace 'mask-on' settings ( $\mathrm{p}=0.036$ by Chi square test of goodness-of-fit).

CONCLUSION: Community-wide mask wearing may contribute to the control of COVID-19 by reducing the amount of emission of infected saliva and respiratory droplets from individuals with subclinical or mild COVID-19.

\section{READING 8 - MODELLING THE POTENTIAL FOR FACE MASK USE BY THE GENERAL PUBLIC TO CURTAIL THE COVID-I9 PANDEMIC}

Eikenberry SE, Mancuso M, Iboi E, Phan T, Eikenberry K, Kuang Y, Kostelich E, Gumel AB. To mask or not to mask: Modeling the potential for face mask use by the general public to curtail the COVID-I9 pandemic. Infect Dis Model. 2020 Apr 21;5:293-308.

\section{URL: doi: I0.1016/j.idm.2020.04.00 I (Free full text). PMID: 32355904; PMCID: PMC7 186508 eCollection 2020.}

Author information:

(1)School of Mathematical and Statistical Sciences, Arizona State University, Tempe, AZ, 85287, USA.

\section{ABSTRACT}

Face mask use by the general public for limiting the spread of the COVID-19 pandemic is controversial, though increasingly recommended, and the potential of this intervention is not well understood.

We develop a compartmental model for assessing the community-wide impact of mask use by the general, asymptomatic public, a portion of which may be asymptomatically infectious.

Model simulations, using data relevant to COVID-19 dynamics in the US states of New York and Washington, suggest that broad adoption of even relatively ineffective face masks may meaningfully reduce community transmission of COVID-19 and decrease peak hospitalisations and deaths.

Moreover, mask use decreases the effective transmission rate in nearly linear proportion to the product of mask effectiveness (as a fraction of potentially infectious contacts blocked) and coverage rate (as a fraction of the general population), while the 
impact on epidemiologic outcomes (death, hospitalisations) is highly nonlinear, indicating masks could synergize with other non-pharmaceutical measures. Notably, masks are found to be useful with respect to both preventing illness in healthy persons and preventing asymptomatic transmission. Hypothetical mask adoption scenarios, for Washington and New York state, suggest that immediate near universal ( 80 percent) adoption of moderately (50 percent) effective masks could prevent on the order of 17-45 percent of projected deaths over two months in New York, while decreasing the peak daily death rate by 34-58 percent, absent other changes in epidemic dynamics. Even very weak masks (20 percent effective) can still be useful if the underlying transmission rate is relatively low or decreasing: In Washington, where baseline transmission is much less intense, 80 percent adoption of such masks could reduce mortality by 24-65 percent (and peak deaths 15-69 percent), compared to 2-9 percent mortality reduction in New York (peak death reduction 9-18 percent).

Our results suggest use of face masks by the general public is potentially of high value in curtailing community transmission and the burden of the pandemic. The community-wide benefits are likely to be greatest when face masks are used in conjunction with other non-pharmaceutical practices (such as social-distancing), and when adoption is nearly universal (nation-wide) and compliance is high.

\section{READING 9 - EFFICACY OF FACE MASKS AND RESPIRATORS USE FOR COMMUNITY, HEALTH CARE WORKSERS AND SICK PATIENTS}

\section{Maclntyre CR(I), Chughtai AA(2). A rapid systematic review of the efficacy of face masks and respirators against coronaviruses and other respiratory transmissible viruses for the community, healthcare workers and sick patients. Int J Nurs Stud. 2020 Apr 30; 1 08: 103629.}

URL: doi: 10.1016/j.ijnurstu.2020.103629.

Online ahead of print.

Author information:

(1)The Kirby Institute, UNSW Medicine, University of New South Wales, Sydney, Australia. Electronic address: r.macintyre@unsw.edu.au. (2)School of Public Health and Community Medicine, UNSW Medicine, University of New South Wales, Room 228, Level 2 Samuels Building, Sydney, Australia. Electronic address: abrar.chughtai@unsw.edu.au.

\section{ABSTRACT}

BACKGROUND: The pandemic of COVID-19 is growing, and a shortage of masks and respirators has been reported globally. Policies of health organisations for healthcare workers are inconsistent, with a change in policy in the US for universal face mask use. The aim of this study was to review the evidence around the efficacy of masks and respirators for healthcare workers, sick patients and the general public.

METHODS: A systematic review of randomised controlled clinical trials on use of respiratory protection by healthcare workers, sick patients and community members was conducted. Articles were searched on Medline and Embase using key search terms.

RESULTS: A total of 19 randomised controlled trials were included in this study - eight in community settings, six in healthcare settings and five as source control. Most of these randomised controlled trials used different interventions and outcome measures. In the community, masks appeared to be effective with and without hand hygiene, and both together are more protective. Randomised controlled trials in health care workers showed that respirators, if worn continually during a shift, were effective but not if worn intermittently. Medical masks were not effective, and cloth masks even less effective. When used by sick patients randomised controlled trials suggested protection of well contacts.

CONCLUSION: The study suggests that community mask use by well people could be beneficial, particularly for COVID-19, where transmission may be pre-symptomatic. The studies of masks as source control also suggest a benefit, and may be important during the COVID-19 pandemic in universal community face mask use as well as in health care settings. Trials in healthcare workers support the use of respirators continuously during a shift. This may prevent health worker infections and deaths from COVID-19, as aerosolisation in the hospital setting has been documented. 


\section{READING 10 - CLOTH MASKS VERSUS MEDICAL MASKS FOR COVID- I 9 PROTECTION}

\section{Szarpak L(I)(2), Smereka J(3)(4), Filipiak KJ(5), Ladny JR(6)(4), Jaguszewski M(7). Cloth masks versus medical masks for Covid- 19 protection. Cardiol J. 2020;27(2):2 18-2 19.}

\section{URL: doi: 10.5603/CJ.a2020.0054 (Free full text). Epub 2020 Apr 14.}

Author information:

(1)Lazarski University, Warsaw, Poland. lukasz.szarpak@gmail.com. (2)Polish Society of Disaster Medicine, Warsaw, Poland. lukasz.szarpak@gmail.com. (3)Department of Emergency Medical Service, Wroclaw Medical University, Wroclaw, Poland. (4)Polish Society of Disaster Medicine, Warsaw, Poland. (5)1st Chair and Department of Cardiology, Medical University of Warsaw, Poland. (6)Department of Emergency Medicine, Medical University of Bialystok, Poland. (7)1st Department of Cardiology, Medical University of Gdansk, Poland.

\section{ABSTRACT}

The five points of note below are reproduced from this Letter to the Editor. Figure 1 shows the results of meta-analysis effectiveness of medical masks versus cloth masks against respiratory infection. Outcomes are clinical respiratory illness (A); influenza like illness (B); and laboratory confirmed viruses (C). $\mathrm{Cl}=$ Clinical interval.

1- "As indicated in the research published by MacIntre et al. [6] in a study on the comparison of the efficacy of cloth masks to medical masks in the context of viral infections in hospital healthcare workers, summarised that cloth masks don't protect as well as medical masks (Fig. 1).

2-Laboratory tests showed the penetration of particles through the cloth masks to be very high (97 percent) compared with medical masks (44 percent). A consequence of the above penetration is also a higher risk of critical care illness, the influenza-like illness is more significant in the cloth mask group than in the medical mask.

3-Moreover, the rate of confirmation of laboratory-confirmed viruses was also much higher for cloth masks than for medical masks or groups that did not wear any mask. In the era of this deficit of masks, another problem arises, to which particular attention should be paid.

4-Most people in all seriously affected areas are reusing their disposable masks. The physical properties of a cloth mask, reuse, the frequency and effectiveness of cleaning, and increased moisture retention, may potentially increase the infection risk, since, as it indicated by Osterholm et al. [7] the virus may survive on the surface of the facemasks. In this context self-contamination through repeated use and improper doffing is possible.

5-Observations during SARS suggested double-masking and other practices increased the risk of infection because of moisture, liquid diffusion and pathogen retention [8].” [See Figure 1]

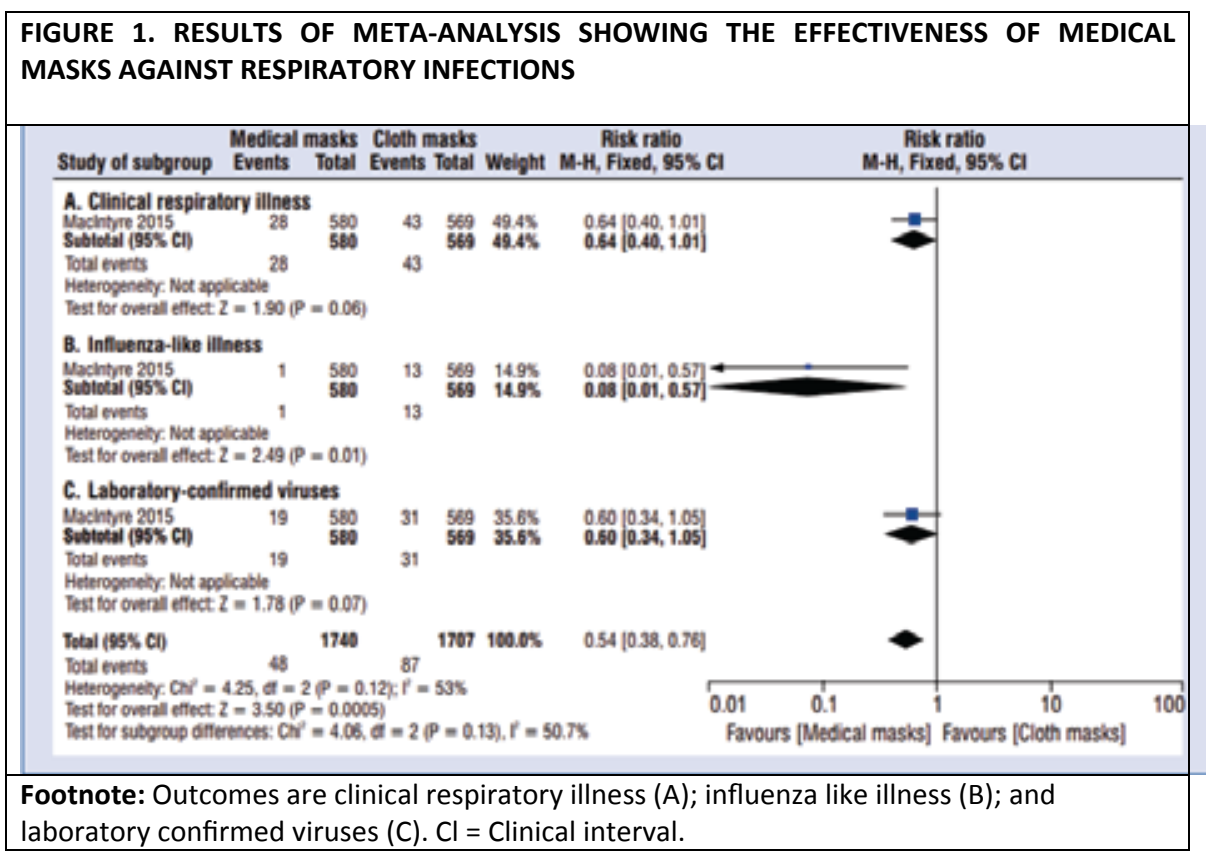




\section{READING I I - COVID- I 9 INFECTIONS - UNDERSTANDING PATHOPHYSIOLOGICAL AND IMMUNOLOGICAL FEATURES}

Tay MZ(I), Poh CM(I), Rénia L(2)(3), MacAry PA(4), Ng LFP(5)(6)(7). The trinity of COVID- I9: immunity, inflammation and intervention.Nat Rev Immunol. 2020 Jun;20(6):363-374.

\section{URL: doi: I 0. I 038/s4 | 577-020-03 | I-8 (Free full text). Epub 2020 Apr 28.}

Author information:

(1)Singapore Immunology Network (SIgN), Agency for Science, Technology and Research (A*STAR), Biopolis, Singapore, Singapore. (2)Singapore Immunology Network (SIgN), Agency for Science, Technology and Research (A*STAR), Biopolis, Singapore, Singapore. renia_laurent@immunol.a-star.edu.sg. (3)Department of Microbiology and Immunology, Yong Loo Lin School of Medicine, National University of Singapore, Singapore, Singapore. renia_laurent@immunol.a-star.edu.sg. (4)Department of Microbiology and Immunology, Yong Loo Lin School of Medicine, National University of Singapore, Singapore, Singapore. micpam@nus.edu.sg. (5)Singapore Immunology Network (SIgN), Agency for Science, Technology and Research (A*STAR), Biopolis, Singapore, Singapore. lisa_ng@immunol.a-star.edu.sg. (6)Department of Biochemistry, Yong Loo Lin School of Medicine, National University of Singapore, Singapore, Singapore. lisa_ng@immunol.a-star.edu.sg. (7)Institute of Infection, Veterinary \& Ecological Sciences, University of Liverpool, Liverpool, UK. lisa_ng@immunol.a-star.edu.sg.

\section{ABSTRACT}

Severe acute respiratory syndrome coronavirus 2 (SARS-CoV-2) is the causative agent of the ongoing coronavirus disease 2019 (COVID-19) pandemic. Alongside investigations into the virology of SARS-CoV-2, understanding the fundamental physiological and immunological processes underlying the clinical manifestations of COVID-19 is vital for the identification and rational design of effective therapies.

Here, we provide an overview of the pathophysiology of SARS-CoV-2 infection. We describe the interaction of SARS-CoV-2 with the immune system and the subsequent contribution of dysfunctional immune responses to disease progression.

From nascent reports describing SARS-CoV-2, we make inferences on the basis of the parallel pathophysiological and immunological features of the other human coronaviruses targeting the lower respiratory tract - severe acute respiratory syndrome coronavirus (SARS-CoV) and Middle East respiratory syndrome coronavirus (MERS-CoV).

Finally, we highlight the implications of these approaches for potential therapeutic interventions that target viral infection and/or immunoregulation.

\section{READING I 2 - COVID- 19 INFECTIONS - EPIDEMIOLOGICAL AND CLINICAL PREDICTORS}

Sun Y(1), Koh V(2)(3), Marimuthu K(2)(3)(4), Ng OT(2)(3)(5), Young B(2)(3)(6), Vasoo S(2)(3), Chan M(2)(3), Lee VJM(I)(7), De PP(3), Barkham T(3)(4), Lin RTP(2), Cook AR(2)(1), Leo YS(2)(3)(5)(4)(1). Epidemiological and Clinical Predictors of COVID- 19. Clin Infect Dis. 2020 Mar 25:ciaa322.

\section{URL: doi: I0.1 093/cid/ciaa322 (Payment required)}

Online ahead of print.

Author information:

(1)Saw Swee Hock School of Public Health, National University of Singapore and National University Health System, Science Drive 2, Singapore. (2)Department of Infectious Diseases, National Centre for Infectious Diseases, Jalan Tan Tock Seng, Singapore. (3)Department of Infectious Diseases, Tan Tock Seng Hospital, Jalan Tan Tock Seng, Singapore. (4)Yong Loo Lin School of Medicine, National University of Singapore and National University Health System, Medical Drive, Singapore. 
(5)Lee Kong Chian School of Medicine, Nanyang Technological University, Mandalay Road, Singapore. (6)Lee Kong Chian School of Medicine, Nanyang Technological University, Singapore. (7)Communicable Disease Division, Ministry of Health, Singapore.

\section{ABSTRACT}

BACKGROUND: Rapid identification of COVID-19 cases, which is crucial to outbreak containment efforts, is challenging due to the lack of pathognomonic symptoms and in settings with limited capacity for specialised nucleic acid-based reverse transcription polymerase chain reaction (PCR) testing.

METHODS: This retrospective case-control study involves subjects (7 to 98 years) presenting at the designated national outbreak screening centre and tertiary care hospital in Singapore for SARS-CoV-2 testing from January 26 to February 16, 2020. COVID-19 STATUS was confirmed by PCR testing of sputum, nasopharyngeal swabs or throat swabs. Demographic, clinical, laboratory and exposure-risk variables ascertainable at presentation were analysed to develop an algorithm for estimating the risk of COVID-19. Model development used Akaike's information criterion in a stepwise fashion to build logistic regression models, which were then translated into prediction scores. Performance was measured using receiver operating characteristics curves, adjusting for over-confidence using leave-out-one cross validation.

RESULTS: The study population included 788 subjects, of whom 54 (6.9 percent) were SARS-CoV-2 positive and 734 (93.1 percent) were SARS-CoV-2 negative. The median age was 34 years and 407 (51.7 percent) were female. Using leave-out-one cross validation, all the models incorporating clinical tests (Models 1, 2 and 3) performed well with areas under the receiver operating characteristics curve (AUC) of $0.91,0.88$ and 0.88 respectively. In comparison, Model 4 had an AUC of 0.65 .

CONCLUSIONS: Rapidly ascertainable clinical and laboratory data could identify individuals at high risk of COVID-19 and enable prioritisation of PCR-testing and containment efforts. Basic laboratory test results were crucial to prediction models.

\section{READING I 3 - COVID- I 9 INFECTION - EPIDEMIOLOGICAL FEATURES AND CLINICAL COURSE OF FIRST I 8 PATIENTS IN SINGAPORE}

Young BE(I)(2)(3), Ong SWX(I)(2), Kalimuddin S(4)(5), Low JG(4)(6), Tan SY(7), Loh J(8), Ng OT(I)(2)(3), Marimuthu K(I)(2)(9), Ang LW(I), Mak TM(I), Lau SK(10), Anderson DE(6), Chan KS(4), Tan TY(6)(7), Ng TY(8), Cui L(I), Said Z(I I), Kurupatham L(I I), Chen MI(I)(I 2), Chan M(I)(2), Vasoo S(I)(2), Wang LF(6), Tan BH(3)(10), Lin RTP(I), Lee VJM(I I)(12), Leo YS(I)(2)(3)(9)(1 2), Lye DC(I)(2)(3)(9); Singapore 2019 Novel Coronavirus Outbreak Research Team. Epidemiologic Features and Clinical Course of Patients Infected With SARS-CoV-2 in Singapore. JAMA. 2020 Mar 3;323(1 5): I 488-94.

\section{URL: doi: 10.100 I/jama.2020.3204 (Free full text).} Online ahead of print.

Author information:

(1)National Centre for Infectious Diseases, Singapore. (2)Tan Tock Seng Hospital, Singapore. (3)Lee Kong Chian School of Medicine, Singapore. (4)Singapore General Hospital, Singapore. (5)Duke-NUS Graduate Medical School, Singapore. (6)Duke-NUS Medical School, Singapore. (7)Changi General Hospital, Singapore. (8)Sengkang General Hospital, Singapore. (9)Yong Loo Lin School of Medicine, Singapore. (10)DSO National Laboratories, Singapore. (11)Ministry of Health, Singapore. (12)Saw Swee Hock School of Public Health, Singapore.

\section{ABSTRACT}

IMPORTANCE: Severe acute respiratory syndrome coronavirus 2 (SARS-CoV-2) emerged in Wuhan, China, in December 2019 and has spread globally with sustained human-to-human transmission outside China. OBJECTIVE: To report the initial experience in Singapore with the epidemiologic investigation of this outbreak, clinical features, and management. 
chain reaction (PCR)-confirmed SARS-CoV-2 infection at four hospitals in Singapore from January 23 to February 3, 2020; final follow-up date was February 25, 2020.

EXPOSURES: Confirmed SARS-CoV-2 infection.

MAIN OUTCOMES AND MEASURES: Clinical, laboratory, and radiologic data were collected, including PCR cycle threshold values from nasopharyngeal swabs and viral shedding in blood, urine, and stool. Clinical course was summarised, including requirement for supplemental oxygen and intensive care and use of empirical treatment with lopinavir-ritonavir.

RESULTS: Among the 18 hospitalised patients with PCR-confirmed SARS-CoV-2 infection (median age, 47 years; 9 [50 percent] women), clinical presentation was an upper respiratory tract infection in 12 (67 percent), and viral shedding from the nasopharynx was prolonged for 7 days or longer among 15 (83 percent). Six individuals (33 percent) required supplemental oxygen; of these, 2 required intensive care. There were no deaths. Virus was detectable in the stool (4/8 [50 percent]) and blood (1/12 [8 percent]) by PCR but not in urine. Five individuals requiring supplemental oxygen were treated with lopinavir-ritonavir. For three of the five patients, fever resolved and supplemental oxygen requirement was reduced within three days, whereas two deteriorated with progressive respiratory failure. Four of the five patients treated with lopinavir-ritonavir developed nausea, vomiting, and/or diarrhoea, and three developed abnormal liver function test results.

CONCLUSIONS AND RELEVANCE: Among the first 18 patients diagnosed with SARS-CoV-2 infection in Singapore, clinical presentation was frequently a mild respiratory tract infection. Some patients required supplemental oxygen and had variable clinical outcomes following treatment with an antiretroviral agent.

\section{READING I4 - COVID- I 9 OUTBREAK - NARRATIVE SYNTHESIS OF PSYCHOLOGICAL IMPACT AND COPING RESPONSES}

\section{Chew QH(I), Wei KC(I), Vasoo S(2), Chua HC(I), Sim K(I). Narrative synthesis of psychological and coping responses towards emerging infectious disease outbreaks in the general population: practical considerations for the COVID- 19 pandemic. Singapore Med J. 2020 Apr 3.}

\section{URL: doi: I0.I I 622/smedj.2020046 (Free full text). Online ahead of print.}

Author information:

(1)Research Department, Institute of Mental Health, Singapore (2)National Centre for Infectious Diseases, Singapore.

\section{ABSTRACT}

INTRODUCTION:

Emerging infectious disease outbreaks, such as the present coronavirus disease 2019 (COVID-19) pandemic, often have a psychological impact on the well-being of the general population, including survivors and caregivers. Our study aimed to synthesise extant literature regarding the combined psychological responses and coping methods used by the general population in past outbreaks.

METHODS: We conducted a narrative synthesis of the published literature over the last two decades with a quality appraisal of included articles that reported both psychological responses and coping strategies within infectious disease outbreaks.

RESULTS: A total of 144 papers were identified from the search, 24 of which were included in the review. Overall, 18 studies examined the psychosocial responses of the general population towards the severe acute respiratory syndrome epidemic, four studies focused on the Ebola epidemic and two studies covered the H1N1 outbreak. Common themes in psychological responses included anxiety/fears, depression, anger, guilt, grief and loss, post-traumatic stress, and stigmatisation, but also a greater sense of empowerment and compassion towards others. Coping strategies adopted included problem-focused coping (seeking alternatives, self- and other-preservation), seeking social support, avoidance, and positive appraisal of the situation. 
CONCLUSION: Amid the range of psychosocial responses seen in past infectious disease outbreaks, practical considerations for the current COVID-19 pandemic need to focus on the individual in the context of the larger social environment, with an emphasis on raising awareness of the range of possible psychosocial responses, access to psychological help, self- care, empowering self-support groups and sustained engagement with updated, reliable information about the outbreak.

\section{READING I 5 - COVID- I9 PATIENTS WITH DIABETES MELLITUS - PRACTICAL MANAGEMENT RECOMMENDATIONS}

\section{Bornstein SR(I), Rubino F(2), Khunti K(3), Mingrone G(4), Hopkins D(5), Birkenfeld AL(6), Boehm B(7), Amiel S(8), Holt RI(9), Skyler JS(1 0), DeVries JH(I I), Renard E(I 2), Eckel RH(I 3), Zimmet P(I4), Alberti KG(I5), Vidal J(16), Geloneze B(I7), Chan JC(18), Ji L(19), Ludwig B(20). Practical recommendations for the management of diabetes in patients with Covid-19. Lancet Diabetes Endocrinol. 2020 Jun;8(6):546-550.}

\section{URL: doi: 10.10 16/S22 I 3-8587(20)30 I 52-2 (Free full text). Epub 2020 Apr 23.}

Author information:

(1)Department of Medicine III, University Hospital Carl Gustav Carus, Dresden, Germany; Paul Langerhans Institute Dresden of the Helmholtz Center Munich, University Hospital Carl Gustav Carus, Dresden, Germany; Department of Diabetes, School of Life Course Science and Medicine, King's College London, London, UK; Department of Endocrinology and Diabetology, University Hospital Zurich, Zurich, Switzerland; Faculty of Medicine, Technische Universität Dresden, Dresden, Germany; German Center for Diabetes Research (DZD e.V.), Neuherberg, Germany. Electronic address: Stefan.Bornstein@uniklinikum-dresden.de. (2)Department of Diabetes, School of Life Course Science and Medicine, King's College London, London, UK; Bariatric and Metabolic Surgery, King's College Hospital, London, UK. (3)Diabetes Research Centre, University of Leicester, Leicester, UK. (4)Department of Diabetes, School of Life Course Science and Medicine, King's College London, London, UK; Fondazione Policlinico Universitario Agostino Gemelli Istituto Di Ricovero e Cura a Carattere Scientifico, Rome, Italy; Department of Internal Medicine, Università Cattolica del Sacro Cuore, Rome, Italy. (5)Department of Diabetes, School of Life Course Science and Medicine, King's College London, London, UK; Institute of Diabetes Endocrinology and Obesity, King's Health Partners, London, UK. (6)Department of Diabetes, School of Life Course Science and Medicine, King's College London, London, UK; German Center for Diabetes Research (DZD e.V.), Neuherberg, Germany; Department of Diabetology, Endocrinology and Nephrology, University Hospital Tübingen, Tübingen, Germany; Institute for Diabetes Research and Metabolic Diseases of the Helmholtz Centre Munich at the University of Tübingen, Tübingen, Germany. (7)Department of Endocrinology, Tan Tock Seng Hospital, Singapore, Singapore; Lee Kong Chian School of Medicine, Nanyang Technological University Singapore, Singapore. (8)Department of Diabetes, School of Life Course Science and Medicine, King's College London, London, UK. (9)Human Development and Health, Faculty of Medicine, University of Southampton, UK. (10)Diabetes Research Institute, University of Miami Miller School of Medicine, Miami, FL, USA. (11)Department of Endocrinology, Amsterdam University Medical Center, University of Amsterdam, Amsterdam, the Netherlands; Profil Institute for Metabolic Research, Neuss, Germany. (12)Montpellier University Hospital and Institute of Functional Genomics, Centre national de la recherche scientifique, INSERM, University of Montpellier, Montpellier, France. (13)Division of Endocrinology, Metabolism, and Diabetes, University of Colorado Anschutz Medical Campus, Aurora, CO, USA. (14)Department of Diabetes, Central Clinical School, Monash University, Melbourne, VIC, Australia. (15)Division of Diabetes, Endocrinology and Metabolism, Imperial College, London, UK. (16)Centro de Investigación Biomédica en Red en Diabetes y Enfermedades Metabólicas Asociadas, Barcelona, Spain. (17)Obesity and Comorbities Research Center, Laboratory of Investigation in Metabolism and Diabetes/Gastrocentro, Universidade de Campinas, Campinas, São Paulo, Brazil. (18)Department of Medicine and Therapeutics, Hong Kong Institute of Diabetes and Obesity and Li Ka Shing Institute of Health Science, The Chinese University of Hong Kong, Prince of Wales Hospital, Hong Kong Special Administrative Region, China. (19)Department of Endocrinology and Metabolism, Peking University People's Hospital, Peking University Diabetes Center, Beijing, China. (20)Department of Medicine III, University Hospital Carl Gustav Carus, Dresden, Germany; Paul Langerhans Institute Dresden of the Helmholtz Center Munich, University Hospital Carl Gustav Carus, Dresden, Germany; Department of Endocrinology and Diabetology, University Hospital Zurich, Zurich, Switzerland; Faculty of Medicine, Technische Universität Dresden, Dresden, Germany; Deutsche Forschungsgemeinschaft-Center for Regenerative Therapies Dresden, Technische Universität Dresden, Dresden, Germany; German Center for Diabetes Research (DZD e.V.), Neuherberg, Germany. 


\section{ABSTRACT}

Diabetes is one of the most important comorbidities linked to the severity of all three known human pathogenic coronavirus infections, including severe acute respiratory syndrome coronavirus 2 .

Patients with diabetes have an increased risk of severe complications including Adult Respiratory Distress Syndrome and multi-organ failure. Depending on the global region, 20-50 percent of patients in the coronavirus disease 2019 (COVID-19) pandemic had diabetes. Given the importance of the link between COVID-19 and diabetes, we have formed an international panel of experts in the field of diabetes and endocrinology to provide some guidance and practical recommendations for the management of diabetes during the pandemic.

We aim to briefly provide insight into potential mechanistic links between the novel coronavirus infection and diabetes, present practical management recommendations, and elaborate on the differential needs of several patient groups.

\section{READING 16 - MEDICATIONS FOR COVID- 9 PATIENTS WITH MUSCULOSKELETAL CONDITIONS}

Tan SHS(1), Hong CC(2), Saha S(2), Murphy D(2), Hui JH(2). Medications in COVID-19 patients: summarizing the current literature from an orthopaedic perspective. Int Orthop. 2020 May 22: I-5.

\section{URL: doi: I 0.1 007/s00264-020-04643-5 (Free full text). Online ahead of print.}

Author information:

(1)Department of Orthopaedic Surgery, National University Health System, National University Hospital, 1E Kent Ridge Road, NUHS Tower Block Level 11, Singapore, 119074, Singapore. sharon_sh_tan@nuhs.edu.sg. (2)Department of Orthopaedic Surgery, National University Health System, National University Hospital, 1E Kent Ridge Road, NUHS Tower Block Level 11, Singapore, 119074, Singapore.

\section{ABSTRACT}

PURPOSE: The review aims to provide a summary of the current literature regarding common medications prescribed in orthopaedic surgery and their potential implications in COVID-19 patients.

METHODS: A systematic review was performed using the PRISMA guidelines. All clinical studies, reviews, consensus and guidelines related to the above medications and COVID-19 were included.

RESULTS: A total of 18 articles were included. The use of analgesia, anti-inflammatories, steroids, anticoagulants, antibiotics, vitamin $\mathrm{B}$, vitamin $\mathrm{C}$ and vitamin $\mathrm{D}$ and their potential impact on COVID-19 patients were reported.

CONCLUSION: Eight main recommendations were derived from the review. Firstly, paracetamol remains the first line of analgesia and antipyretic.

Secondly, there is no need to avoid NSAIDs for COVID-19 patients.

Thirdly, opioids have the potential for immunosuppression in addition to respiratory depression and, therefore, should be prescribed with care in COVID-19 patients.

Fourthly, patients with conditions where steroids are proven to be efficacious can continue to receive their steroids; otherwise, systemic steroids are not recommended for COVID-19 patients.

Fifthly, orthopaedic surgeons following up on COVID-19 patients who are using steroids should continue to follow them up for possible avascular necrosis.

Sixthly, whenever possible, oral anticoagulation should be converted to parental heparin.

Seventhly, common orthopaedic antibiotics including penicillin and clindamycin are safe to continue for COVID-19 patients. However, for COVID-19 patients, the antibiotics can potentially be switched to macrolides and tetracyclines if the organisms are sensitive.

Lastly, prescription for vitamins $\mathrm{B}, \mathrm{C}$ and $\mathrm{D}$ should continue as per usual clinical practice. 


\title{
READING 17 - COVID- 19 OUTBREAK - STAFF TEMPERATURE AND SICKNESS SURVEILLANCE
}

\author{
Htun HL(I), Lim DW(I)(2), Kyaw WM(I), Loh WJ(I), Lee LT(2), Ang B(3), Chow A(I). \\ Responding to the COVID- 19 outbreak in Singapore: Staff Protection and Staff \\ Temperature and Sickness Surveillance Systems.Clin Infect Dis. 2020 Apr 2 I :ciaa468.
}

URL: doi: 10.1093/cid/ciaa468 (Free full text). Online ahead of print.

Author information:

(1)Department of Clinical Epidemiology, Office of Clinical Epidemiology, Analytics, and Knowledge (OCEAN), Tan Tock Seng Hospital, Singapore. (2)Occupational Health Services, Tan Tock Seng Hospital, Singapore. (3)Department of Infectious Diseases, Tan Tock Seng Hospital, Singapore.

\section{ABSTRACT}

BACKGROUND: Coronavirus disease 2019 (Covid-19) is an emerging infectious disease caused by novel coronavirus (SARS-CoV-2), and first reported in Wuhan, China, in December 2019. Since the severe acute respiratory syndrome (SARS) outbreak in 2003, Tan Tock Seng Hospital (TTSH) in Singapore has routinely fit-tested staff for high filtration N95 respirators, and established web-based staff surveillance systems. The routine systems were enhanced in response to Singapore's first imported COVID-19 case on January 23,2020. METHODS: We conducted a cross-sectional study, from January 23,2020 to February 23,2020, among healthcare workers to evaluate the effectiveness of the staff protection and surveillance strategy in TTSH, a 1600-bed multidisciplinary acute-care hospital co-located with the 330-bed National Centre for Infectious Diseases (NCID). As of February 23,2020, TTSH/NCID has managed 76 percent of confirmed COVID-19 cases in Singapore. The hospital adopted a multi-pronged approach to protect and monitor staff with potential COVID-19 exposures:(1) Risk-based personal protective equipment, (2) Staff fever and sickness surveillance, and (3) Enhanced medical surveillance of unwell staff. RESULTS: A total of 10,583 staff were placed on hospital-wide fever and sickness surveillance, with 1,524 frontline staff working in COVID-19 areas under close surveillance. Among frontline staff, a median of eight staff illness episodes was seen per day, and almost 10 percent $(n=29)$ resulted in hospitalisation. None of the staff was found to be infected with COVID-19. CONCLUSIONS: A robust staff protection and health surveillance system that is routinely implemented during non-outbreak periods and enhanced during the COVID-19 outbreak is effective in protecting frontline staff from the infection.

\section{READING I 8 - COVID-I9 CASE DETECTION AND SEGREGATION OF SUSPECT CASES IN EMERGENCY DEPARTMENT}

Wee LE(I)(2), Fua TP(3), Chua YY(2), Ho AFW(3), Sim XYJ(2)(4), Conceicao EP(4), Venkatachalam I(2)(4), Tan KB(3), Tan BH(2). Containing COVID- 19 in the Emergency Department: The Role of Improved Case Detection and Segregation of Suspect Cases. . Acad Emerg Med. 2020 May;27(5):379-387.

\section{URL:doi: I 0.1 I I I/acem. I 3984 (Free full text).} Epub 2020 May II.

Author information:

(1)From the, Singhealth Infectious Diseases Residency, Singapore. (2)the, Department of Infectious Diseases, Singapore General Hospital, Singapore. (3)the, Department of Emergency Medicine, Singapore General Hospital, Singapore. (4)and the, Department of Infection Prevention and Epidemiology, Singapore General Hospital, Singapore.

\section{ABSTRACT}

OBJECTIVES: Patients with COVID-19 may present with respiratory syndromes indistinguishable from common viruses. This poses a challenge for early detection during triage in the emergency department (ED). Over a three-month period, our ED aimed to minimise nosocomial transmission by using broader suspect case criteria for better detection and using appropriate personal protective equipment (PPE) for health care workers (HCWs). METHODS: All ED admissions with respiratory syndromes over a three-month period were tested for COVID-19. The sensitivity and specificity of screening criteria in detecting COVID-19 were assessed. A risk-stratified approach was adopted for PPE usage in the ED, based on 
high-risk "fever areas" and lower-risk zones. When a case of COVID-19 was confirmed, surveillance was conducted for potentially exposed patients and HCWs. RESULTS: A total of 1,841 cases presenting with respiratory syndromes required admission over the study period. Among these, 70 cases of COVID-19 were subsequently confirmed. The majority (84.2 percent, 59/70) were detected at ED triage because they fulfilled suspect case criteria. Of these, 34 met the official screening criteria; an additional 25 were detected by the broader internal screening criteria. Over the 12-week period, the cumulative sensitivity of internal screening criteria was 84.3 percent ( 95 percent confidence interval $[\mathrm{CI}]=73.6$ percent to 91.9 percent), whereas the sensitivity of the official screening criteria was 48.6 percent ( 95 percent $\mathrm{CI}=36.4$ percent to 60.8 percent). Given the broadened internal criteria, the preexisting ED "fever area" was insufficient and had to be expanded. However, there were no cases of nosocomial transmission from intra-ED exposure, despite extensive surveillance. CONCLUSION: Frontline physicians need to be given leeway to decide on the disposition of cases based on clinical suspicion during an ongoing outbreak of COVID-19. If a broader criterion is used at ED triage, ED facilities and isolation facilities need to be readied to accommodate a surge of suspect cases. Usage of appropriate PPE is essential in minimizing nosocomial transmission.

\title{
READING 19 - COVID-I9 RETROSPCTIVE CASE SERIES FROM PRIMARY CARE - EARLY CLINICAL IDENTIFICATION OF CASES
}

\section{Sim SZ(I), Teo SH(I), Kong JW(I), Lim Z(I), Ng MYK(I), Tang WE(I). Covid- 19 in Singapore - a case series} from primary care. Singapore Med J. 2020 May 27.

URL: doi: 10.1 1622/smedj.2020082 (Free full text).

Online ahead of print.

\section{Author information: \\ (I)National Health Group Polyclinics, Singapore.}

\section{ABSTRACT}

As the coronavirus disease 2019 (COVID-19) pandemic worsens, early case detection is vital to limiting community spread. We describe our experiences with four COVID-19 cases at the polyclinics in January and February 2020. This retrospective case series highlights the challenges primary care clinicians face in the early identification of suspect cases based on clinical criteria only. To improve case detection, clinicians can sharpen their clinical acumen by keeping abreast with the latest COVID-19 developments and by maintaining a high state of vigilance.

\section{READING 20 - COVID- I 9 INFECTION IN PRIMARY CARE - FRAMEWORK FOR EARLY IDENTIFICATION AND ISOLATION}

Lim WH(I), Wong WM(2). COVID- 19: Notes From the Front Line, Singapore's Primary Health Care Perspective. Ann Fam Med. 2020 May; 18(3):259-26I.

\section{URL: doi: I0.1370/afm.2539 (Free full text).}

\author{
Author information: \\ (I)Division of Primary Care, Raffles Medical Group, Singapore \\ lim_weihan@rafflesmedical.com. \\ (2)Division of Primary Care, Raffles Medical Group, Singapore.
}

\section{ABSTRACT}

Coronavirus disease 2019 (COVID-19) is a rapidly progressing global pandemic against which nations are struggling for containment. Singapore is known to have promptly instituted aggressive public health and containment measures. A key pillar sustaining this is the response of its primary health care network. It is important for health care systems worldwide to recognise the value of a strong coordinated response to this crisis from a primary health perspective. There are best practices for early isolation and containment of suspect cases while protecting health care workers and limiting cross infections that are 
transferable across nations. We describe our framework for how our primary care clinics respond to this pandemic in the hope others may find solutions to their unique needs. Moving forward, there is a pressing need for more studies to enhance our understanding of the response of primary care during these public health crises. 